\title{
L-mode radiative plasma edge studies for model validation in ASDEX Upgrade and JET
}

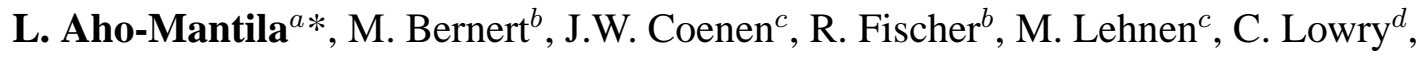 \\ S. Marsen ${ }^{e}$, K. McCormick ${ }^{b}$, H.W. Müller ${ }^{b}$, B. Sieglin ${ }^{b}$, M.F. Stamp ${ }^{f}$, M. Wischmeier ${ }^{b}$, \\ X. Bonnin ${ }^{g}$, D.P. Coster ${ }^{b}$, D. Reiter ${ }^{c}$, S. Brezinsek ${ }^{c}$ and the ASDEX Upgrade Team and the \\ JET-EFDA Contributors \\ JET-EFDA, Culham Science Centre, Abingdon, OX14 3DB, UK \\ ${ }^{a}$ VTT Technical Research Centre of Finland, FI-02044 VTT, Finland \\ ${ }^{b}$ Max-Planck Institut für Plasmaphysik, D-85748 Garching, Germany \\ ${ }^{c}$ Energie- und Klimaforschung IEK-4, FZJ, EURATOM Assoc., TEC, 52425 Jülich, Germany \\ ${ }^{d}$ EFDA JET CSU, Culham Science Centre, OX14 3DB, Abingdon, UK \\ ${ }^{e}$ Max-Planck-Institut für Plasmaphysik, Teilinsitut Greifswald, D-17491 Greifswald, Germany \\ ${ }^{f}$ Culham Centre for Fusion Energy, EURATOM-CCFE Association, Abingdon, UK \\ ${ }^{g}$ LSPM - CNRS, Université Paris 13, F-93430 Villetaneuse, France \\ +See App. of F. Romanelli et al., Proc. of the 23rd IAEA Fusion Energy Conf. 2010, Daejeon, Korea
}

\begin{abstract}
The presently favoured option for reactor power handling combines metallic plasma-facing components and impurity seeding to achieve highly radiative scrape-off layer and divertor plasmas. It is uncertain whether tolerable divertor power loads will be obtained in this scenario, necessitating the development of predictive modelling tools. L-mode experiments with $\mathrm{N}_{2}$ seeding have been conducted at both ASDEX Upgrade and JET for benchmarking the critically important impurity radiation models in edge fluid codes. In both machines, a roll-over in ion fluxes and a reduction in target power load is observed first in the inner divertor. The outer divertor evolves from low-recycling to conduction-limited conditions once a reduction of inner target particle load is observed. First comparisons between SOLPS5.0 simulations and $\mathrm{N}_{2}$ seeded experiment support the observed in-out asymmetry in the effect of seeding.
\end{abstract}


PACS: 52.25.Vy, 52.30.Ex, 52.55Fa, 52.55.Rk, 52.65.Kj

PSI-20 keywords: Power exhaust, Impurity seeding, ASDEX-Upgrade, JET-ILW, B2/EIRENE

*Corresponding author address: P.O. Box 1000, FI-02015 VTT

*Corresponding author E-mail: leena.aho-mantila@vtt.fi

Presenting author: Leena Aho-Mantila

Presenting author E-mail: leena.aho-mantila@vtt.fi 


\section{Introduction}

2D multifluid code packages, such as SOLPS [1], EDGE2D [2] and UEDGE [3], are extensively used for modelling the scrape-off layer, SOL, and divertor plasmas in both existing and future devices. In recent years, their capability to reproduce experimental observations has been questioned, particularly for plasmas with at least one of the divertor legs approaching detachment (see e.g. $[4,5]$ and references therein). Partial divertor detachment is mandatory for achieving tolerable target power loads in a reactor. However, the majority of the power has to be first exhausted by radiation in the edge plasma, which in a full-metal device requires injection of external impurities. This necessitates a separate, dedicated validation of impurity radiation models, for the purpose of predictive reactor design studies.

In this paper, we outline an effort to validate the incremental effects of seeded impurities in the edge code packages, taking into account the presently limited capabilities of the codes to model strongly recombining divertor regimes. The studies focus on two all-metal divertor tokamaks, ASDEX Upgrade and JET, and on low-density L-mode plasmas, in which at least one of the divertor legs is in a low-recycling regime. In the full-W ASDEX Upgrade, a good consistency between various divertor diagnostics has been observed for such conditions in the absence of seeding $[6,7]$, suggesting that these divertor conditions are simple enough to be reproduced by the fluid code packages. The experimental results from $\mathrm{N}_{2}$ seeded discharges are discussed in Section 2, followed by first qualitative modelling studies in Section 3.

\section{L-mode $\mathrm{N}_{2}$ seeding experiments in ASDEX Upgrade and JET}

We summarize in this section the experimental results from $\mathrm{N}_{2}$ seeding into low-density, lowersingle-null L-mode discharges in ASDEX Upgrade and JET. The selection of data has been limited by requirements of model validation, i.e. a sufficient diagnostic coverage of the SOL and divertor regions must be available for the investigated discharges. We present results from discharge \#25569 in ASDEX Upgrade, and from the first L-mode $\mathrm{N}_{2}$ seeding experiment (\#82291- 
9) in JET with the ITER-like wall (see also Ohmic experiment in [8]).

Figure 1 summarizes the main differences between the experimental setups in the two devices. The heating powers were 0.7 MW ECRH and 0.5 MW ohmic in ASDEX Upgrade, compared to 1.1 MW NBI and 1.65 MW ohmic in JET. Line-averaged densities were feedbackcontrolled by the $D_{2}$ puff at $\bar{n}_{e}=3.6 \times 10^{19} \mathrm{~m}^{-3}$ and $\bar{n}_{\mathrm{e}}=3.7 \times 10^{19} \mathrm{~m}^{-2}$ respectively, yielding in both machines low-recycling conditions in the outer divertor $\left(T_{\mathrm{e}}=25-35 \mathrm{eV}\right)$ and high-recycling conditions in the inner divertor $\left(T_{\mathrm{e}}=5-8 \mathrm{eV}\right)$. An open divertor geometry, with outer strike point on the horizontal tile, was used in JET compared to the closed divertor geometry in ASDEX Upgrade. Injection of $\mathrm{N}_{2}$ was made from the dome in ASDEX Upgrade and from the outer divertor SOL in JET, as shown in Figure 1.

Strong $\mathrm{N}_{2}$ puffs were made in ASDEX Upgrade in order to achieve a highly radiative edge plasma with significantly reduced divertor power loads, see Figure 2. Due to the short duration and high level of $\mathrm{N}_{2}$ injection rate, these puffs led to a time-dependent response of the plasma, as visible in the oscillating behaviour of the effective core ion charge, $\mathrm{Z}_{\mathrm{eff}}$. A significant perturbation of the plasma conditions was measured during the $\mathrm{N}_{2}$ injections, with $\mathrm{Z}_{\text {eff }}$ increasing by $40 \%$ during the first two puffs. The decay times of $\mathrm{Z}_{\text {eff }}$ appear not to depend on the amount of $\mathrm{N}_{2}$ injected in each puff, and the plasma recovers from the $\mathrm{N}_{2}$ puff within the $0.3 \mathrm{~s}$ between the first and second puff. Up to $90 \%$ radiative power fractions were measured by the bolometers but, because of possible overestimation in the presence of ECRH in that experimental campaign, this measurement is considered uncertain and will be verified in future experiments.

The ASDEX Upgrade discharge serves to characterize what happens in the two divertor legs in the presence of strong $\mathrm{N}_{2}$ puffs. Figure 3 shows the divertor conditions measured by the flush-mounted Langmuir probes. The data are extracted from an $0.1 \mathrm{~s}$ interval at the beginning of the first puff (no $\mathrm{N}_{2}$ ), and at the end of the first $(1.5 \mathrm{E} 22 \mathrm{e} / \mathrm{s})$, second $(1.0 \mathrm{E} 22 \mathrm{e} / \mathrm{s})$ and third puff $(0.6 \mathrm{E} 22 \mathrm{e} / \mathrm{s})$. Injection of $\mathrm{N}_{2}$ cools the inner divertor, reduces the ion fluxes, $\Gamma_{\|}$, at the inner strike point and shifts the peak $\Gamma_{\|}$towards the outer SOL. However, the inner target does not resume the conditions measured prior to the $\mathrm{N}_{2}$ puffs at any later point in the discharge, even though the core $\mathrm{Z}_{\text {eff }}$ resumes the non-seeded level at the beginning of the second puff. This suggest a longer legacy effect of impurities in the divertor compared to core plasma regions. The 
situation is different in the outer divertor, where the same unseeded plasma conditions are measured prior to each $\mathrm{N}_{2}$ puff. A notable reduction of $T_{\mathrm{e}}$ is observed after the first puff, whereas practically no change is observed after the third puff, despite the $20 \%$ increase in $\mathrm{Z}_{\text {eff }}$ shown in Figure 2. This indicates a strong in-out asymmetry in the effect of $\mathrm{N}_{2}$ seeding on the divertor plasma. The observation is supported by measurements made using an IR camera, showing a suppression of power load in the inner divertor with simultaneously negligible changes in the outer divertor.

Unlike in ASDEX Upgrade, the seeding in the JET experiment was done at a constant level throughout the $10 \mathrm{~s}$ flattop, such that steady conditions with respect to $\mathrm{Z}_{\text {eff }}$ and radiated power were obtained at the end of each discharge. Furthermore, the $\mathrm{N}_{2}$ seeding rates, $\Phi_{\mathrm{N}}$, spanned a wider range than in ASDEX Upgrade. Figure 4 shows selected measurements at the end of each JET discharge, as a function of $\Phi_{\mathrm{N}}$. First, one notes an approximately linear increase of $\mathrm{Z}_{\text {eff }}$ with $\Phi_{\mathrm{N}}$. At the lowest seeding rates, radiation increases rapidly in the plasma regions outside the separatrix, whereas core radiation increases linearly with $\mathrm{Z}_{\mathrm{eff}}$, as expected from simple models [9]. SOL plasma is the dominant region of radiation at seeding rates lower than $\Phi_{\mathrm{N}}=7.5 \times 10^{21} \mathrm{e} / \mathrm{s}$, at which level the total radiated power, $\mathrm{P}_{\text {rad,tot }}$, reaches an apparent maximum of $60 \%$ of the total input power. At seeding rates higher than this, only minor changes are observed in $\mathrm{P}_{\text {rad,tot }}$. The main region of radiation appears, however, to move from the edge towards the core plasma.

We note that prior to the saturation of $\mathrm{P}_{\text {rad,tot }}$, the outer divertor cools down to an apparent minimum peak temperature of $8 \mathrm{eV}$, and a roll-over in $\mathrm{W}$ sputtering yield is observed [10]. Furthermore, a strong increase in $D_{2}$ puffing rate with $\Phi_{N}$ is required for a constant $\bar{n}_{e}$, indicating that $\mathrm{N}_{2}$ seeding reduces the deuterium fuelling efficiency (see also H-mode studies in [11]). After saturation of $\mathrm{P}_{\text {rad,tot }}$, further increases in $\Phi_{\mathrm{N}}$ lead to a roll-over in the outer target particle load, $\Gamma_{\text {tot }}$. In accordance, a reduction in the outer target power load is observed throughout the discharge series. At the inner target, temperatures around 5-8 eV are measured in each discharge, and a roll-over in ion flux occurs at a lower $\mathrm{N}_{2}$ seeding rate $\left(\Phi_{\mathrm{N}}=5 \times 10^{21} \mathrm{e} / \mathrm{s}\right)$ compared to the outer divertor, before saturation of $\mathrm{P}_{\text {rad,tot. }}$. From the observed relatively high divertor temperatures and suppressed target sputtering yields we conclude that the measured 
changes in radiation, particularly in the core, are mainly due to $\mathrm{N}$ impurities, as opposed to $\mathrm{W}$ or D.

We note some key similarities between the two experiments. First, the outer divertor appears to remain in a low-recycling regime for increases in $\mathrm{Z}_{\text {eff }}$ up to $20 \%$ in both devices. This level of impurity seeding is, however, sufficient to significantly perturb the conditions in the inner divertor. Namely, strong reductions in inner target particle and power loads are observed. The outer divertor evolves from low-recycling to conduction-limited regime once a reduction in the inner target particle load, $\Gamma_{\text {tot,IT, }}$ is observed. In JET, the particle load at the outer target,

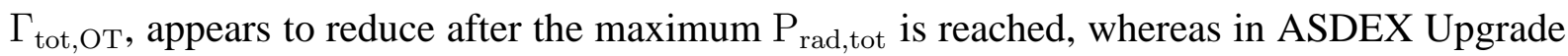
no reduction of $\Gamma_{\text {tot,OT }}$ was observed for the investigated seeding levels.

\section{SOLPS5.0 simulations}

The SOLPS5.0 code package was chosen as subject for a detailed model validation against impurity seeded plasmas in full-metal devices. We limit the modelling studies in this paper to ASDEX Upgrade simulations and to the range of $\Phi_{\mathrm{N}}$ in which the outer divertor cools down without significant reduction in $\Gamma_{\|}$. Within this range of seeding levels, no major changes were observed in the measured upstream conditions, which allows us to use the separatrix density as a constant input in the simulations, as a first approximation. We note, however, that in the JET experiment significant changes were observed in the plasma pedestal at higher levels of $\Phi_{\mathrm{N}}$, which likely has an impact on the observed roll-over in the outer target particle load.

The simulations for ASDEX Upgrade were performed with currents and cross-field drifts $(\mathbf{E} \times \mathbf{B}$, diamagnetic) activated and the 2D plasma fluid code B2.5 iteratively coupled to the 3D Monte Carlo neutrals code Eirene (1999 version). Anomalous transport was described by radially varying transport coefficients which, together with the upstream separatrix density, were adjusted to achieve the best possible agreement with the measured low-recycling conditions in the outer divertor, in the absence of seeding (similar to $[6,7]$ ). Simultaneously, agreement with the measured temperature and density profiles at the outer midplane was requested (within measurement uncertainties), yielding high-recycling inner divertor conditions in the simulations, with density a factor of 2 higher than in the ASDEX Upgrade experiment. The wall was 
specified as $\mathrm{W}$ assuming no erosion, apart from the inner divertor, which was specified as $\mathrm{C}$ to represent the typical residual impurity layers. The seeding was described by a thermal source of $\mathrm{N}$ atoms with cosine angular distribution, launched from the divertor dome. For comparison with JET, we investigated also the effect of changing the seeding location to the outer divertor SOL in the ASDEX Upgrade simulations. The ionization and recombination rates for $\mathrm{N}$ were taken from the ADAS database, and full recycling of $\mathrm{N}$ was assumed.

Figure 5 shows the modelled steady-state values of peak target $T_{\mathrm{e}}$ and $\Gamma_{\text {tot }}$ for the two divertors, as a function of $\mathrm{N}_{2}$ injection rate. Injection of $\mathrm{N}_{2}$ is observed to first cool the inner divertor, with a simultaneous shifting of the peak particle load towards the outer SOL. $\Gamma_{\text {tot,IT }}$ remains constant until the temperature drops below $2 \mathrm{eV}$, after which a drop in $\Gamma_{\text {tot,IT }}$ is observed in the simulation. As $\Gamma_{\text {tot,IT }}$ is reduced, temperature at the outer target decreases rapidly as a function of $\Phi_{\mathrm{N}}$, whereas a relatively small reduction is modelled for $\Gamma_{\text {tot,OT. }} \Gamma_{\text {tot,OT }}$ drops only after the outer target $T_{\mathrm{e}}$ is reduced below $5 \mathrm{eV}$, i.e. at significantly higher $\Phi_{\mathrm{N}}$ compared to the inner target. The results at low $\Phi_{\mathrm{N}}$ are practically unchanged when moving the puffing location into the outer target SOL.

The simulations support the experimental observations, as significant changes are simulated for the inner divertor while the outer divertor is simultaneously in a low-recycling regime. This indicates that the inner divertor conditions are more sensitive to $\mathrm{N}$ impurities, which may determine the overall effect of seeding. This has potentially major influences for model validation, as the plasma conditions are typically less well understood in the inner compared to the outer divertor. Detailed comparisons between the modelled inner divertor solutions and multiple diagnostic measurements will, thus, be needed in the future.

\section{Summary}

Injection of $\mathrm{N}_{2}$ into low-density L-mode plasmas with constant line-averaged density was observed to significantly reduce the particle and power loads at the divertor targets in both ASDEX Upgrade and JET. A reduction in particle load was observed first at the inner target, while the outer divertor remained in a low-recycling regime for increases of $\mathrm{Z}_{\text {eff }}$ up to $20 \%$. A transition to a conduction-limited outer divertor regime was observed in both devices after the reduction 
of inner target particle load. A subsequent reduction of outer target particle load was observed only in the JET experiment, in which the highest $\mathrm{N}_{2}$ seeding levels yielded a maximum radiated power fraction of $60 \%$. SOLPS5.0 simulations agreed qualitatively with the observed in-out asymmetry in the effect of seeding, indicating no sensitivity of the divertor response to the location of the $\mathrm{N}_{2}$ source, at least regarding the steady-state solutions for the closed divertor geometry of ASDEX Upgrade.

\section{Acknowledgements}

This work was supported by EURATOM and carried out within the framework of the European Fusion Development Agreement. The views and opinions expressed herein do not necessarily

reflect those of the European Commission. The work was partially funded by the Academy of Finland project No. 134930. 


\section{References}

[1] Schneider Ret al, 2006 Contrib. Plasma Phys. 46(1-2) 3-191

[2] Taroni A et al, 1992 Contributions to Plasma Physics 32(3-4) 438-443

[3] Rognlien T D et al, 1994 Contributions to Plasma Physics 34(2-3) 362-367

[4] Wischmeier M et al, 2011 Journal of Nuclear Materials 415(1, Supplement) S523 - S529

[5] Chankin A V et al, 2006 Plasma Phys. Control. Fus. 48 839-868

[6] Aho-Mantila L, 2011 Divertor plasma conditions and their effect on carbon migration in the ASDEX Upgrade tokamak. Ph.D. thesis, Aalto University School of Science

[7] Aho-Mantila L et al Outer divertor of ASDEX Upgrade in low-density L-mode discharges in forward and reversed magnetic field Part I: Comparison between measured plasma conditions and SOLPS5.0 code calculations. Submitted to Nuclear Fusion

[8] Oberkofler M, 2012 these proceedings (O-27)

[9] Kallenbach A et al, 2011 Journal of Nuclear Materials 415(1, Supplement) S19 - S26

[10] van Rooij G, 2012 these proceedings (I-3)

[11] Maddison G et al, 2011 Nuclear Fusion 51(4) 042001 


\section{Figures}

Figure 1: Plasma configurations and $\mathrm{N}_{2}$ injection locations in (A) the ASDEX Upgrade experiment and (B) the JET experiment.

Figure 2: Time traces of $\bar{n}_{\mathrm{e}}$ (top) and $\mathrm{Z}_{\text {eff }}$ (middle), measured along a central line-of-sight, and $\mathrm{N}_{2}$ injection rate (bottom) in the ASDEX Upgrade discharge \#25569.

Figure 3: Plasma temperatures and densities measured by the Langmuir probes along the inner (left) and outer (right) targets in ASDEX Upgrade discharge \#25569, after various levels of $\mathrm{N}_{2}$ injection have been applied.

Figure 4: Measured data at the end of each discharge in the JET L-mode $\mathrm{N}_{2}$ seeding experiment (\#82291,\#82293-6, \#82299). Top: $Z_{\text {eff }}$ and $D_{2}$ injection level. Upper middle: Power load measured at the outer target by an IR camera and radiated power fractions in various regions, calculated from 2D tomography of bolometer measurements, with core and edge radiation distinguished by the $\rho=0.98$ flux surface. Lower middle: Peak temperature at the outer target and ion fluxes integrated along the two targets, as measured by the Langmuir probes during a strike point scan (the first data point without $\mathrm{N}_{2}$ injection is taken from a reference discharge \#81603). Bottom: measured $\mathrm{W}$ emission at the outer strike point.

Figure 5: Peak temperatures and particle loads along the two divertor targets in ASDEX Upgrade, as modelled by SOLPS5.0 for various levels of $\mathrm{N}$ injection. The red circles and blue dots refer to $\mathrm{N}$ injection from the outer target and dome, respectively. 

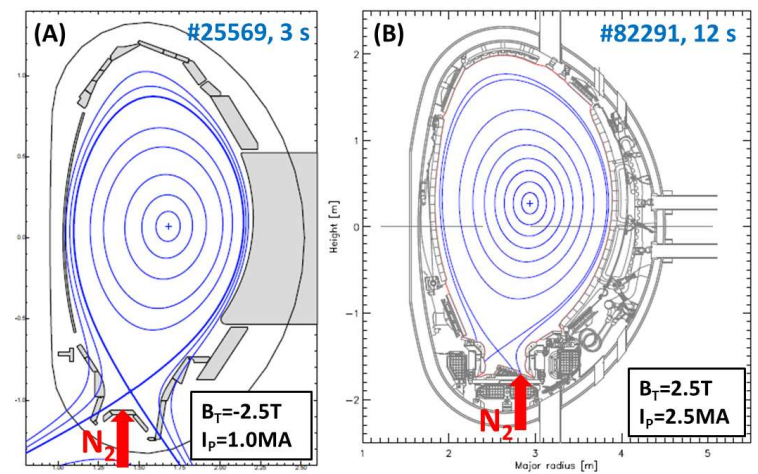

Figure 1 


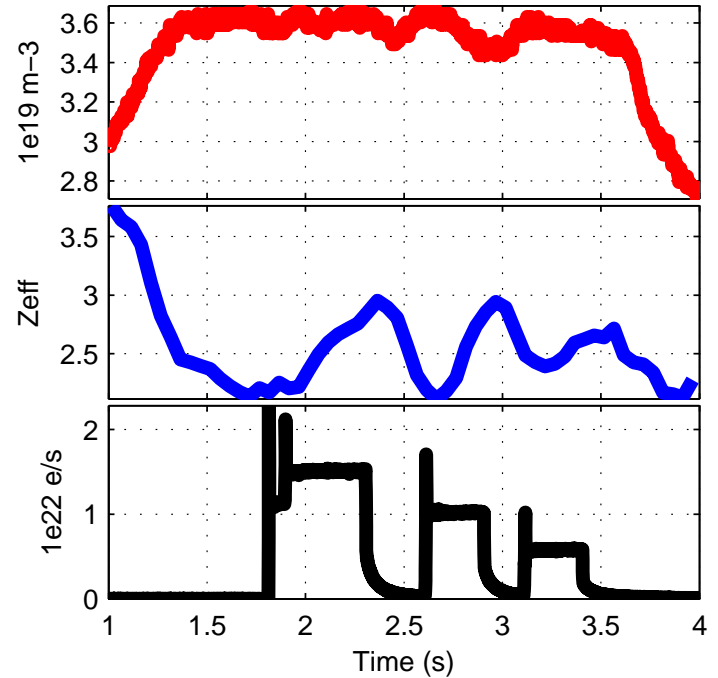

Figure 2 


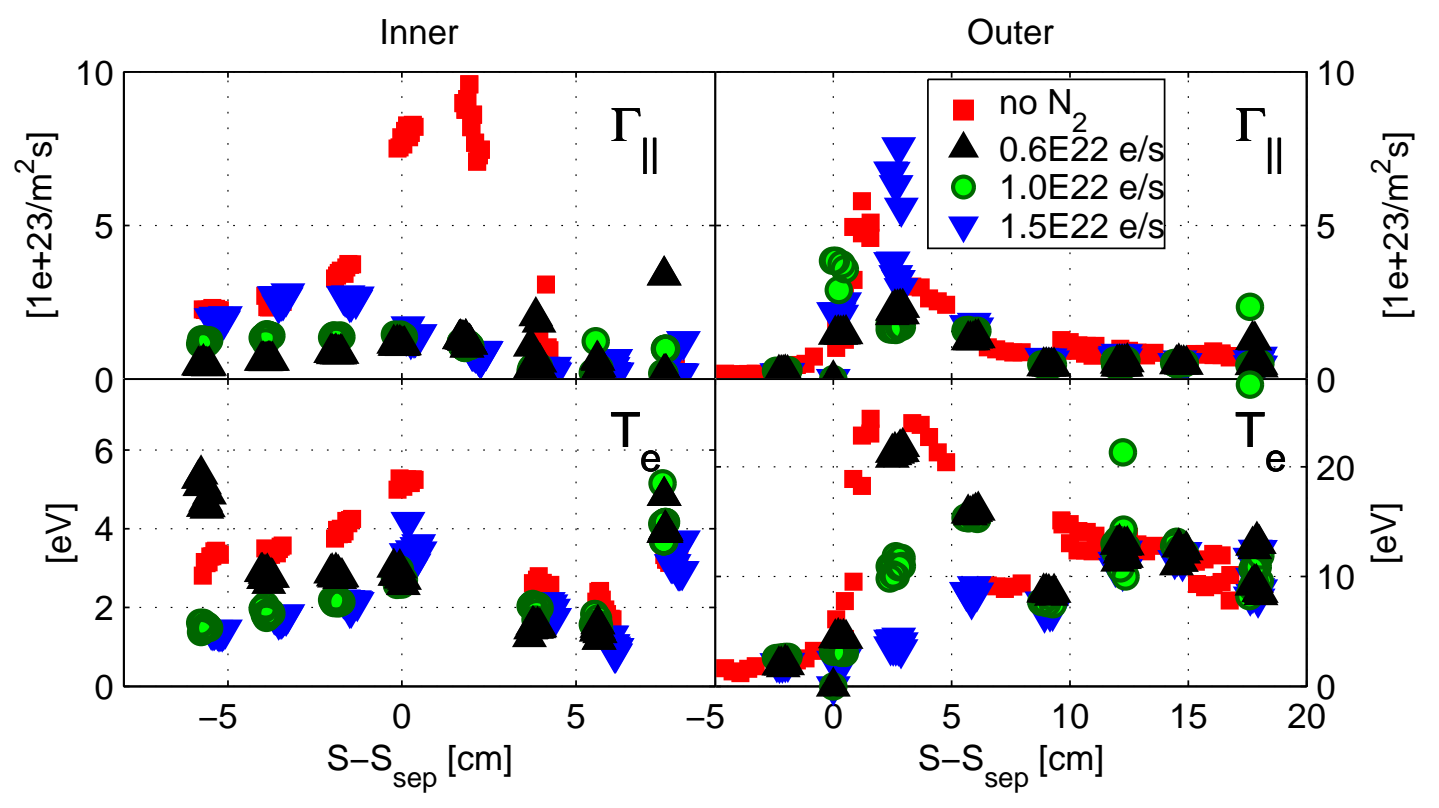

Figure 3 

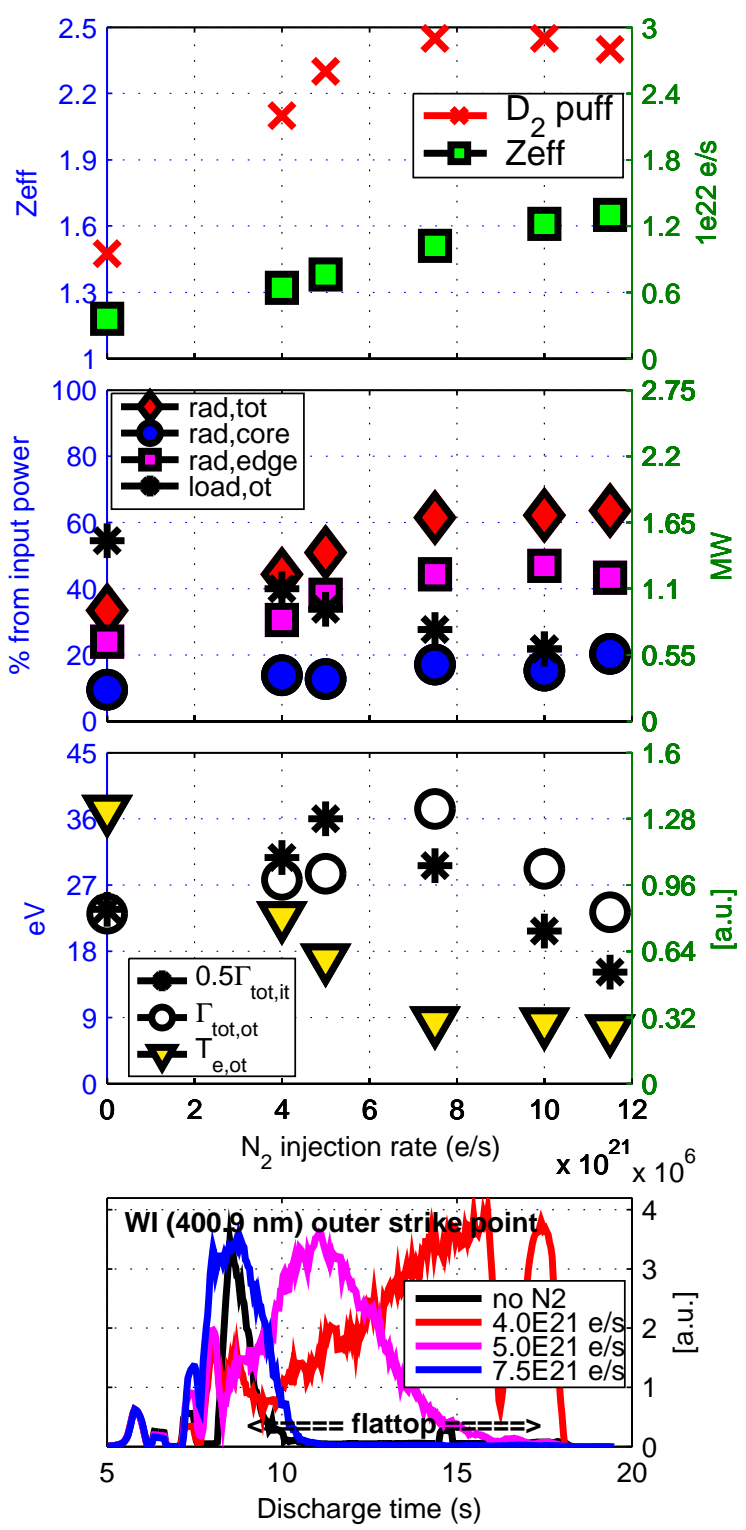

Figure 4 

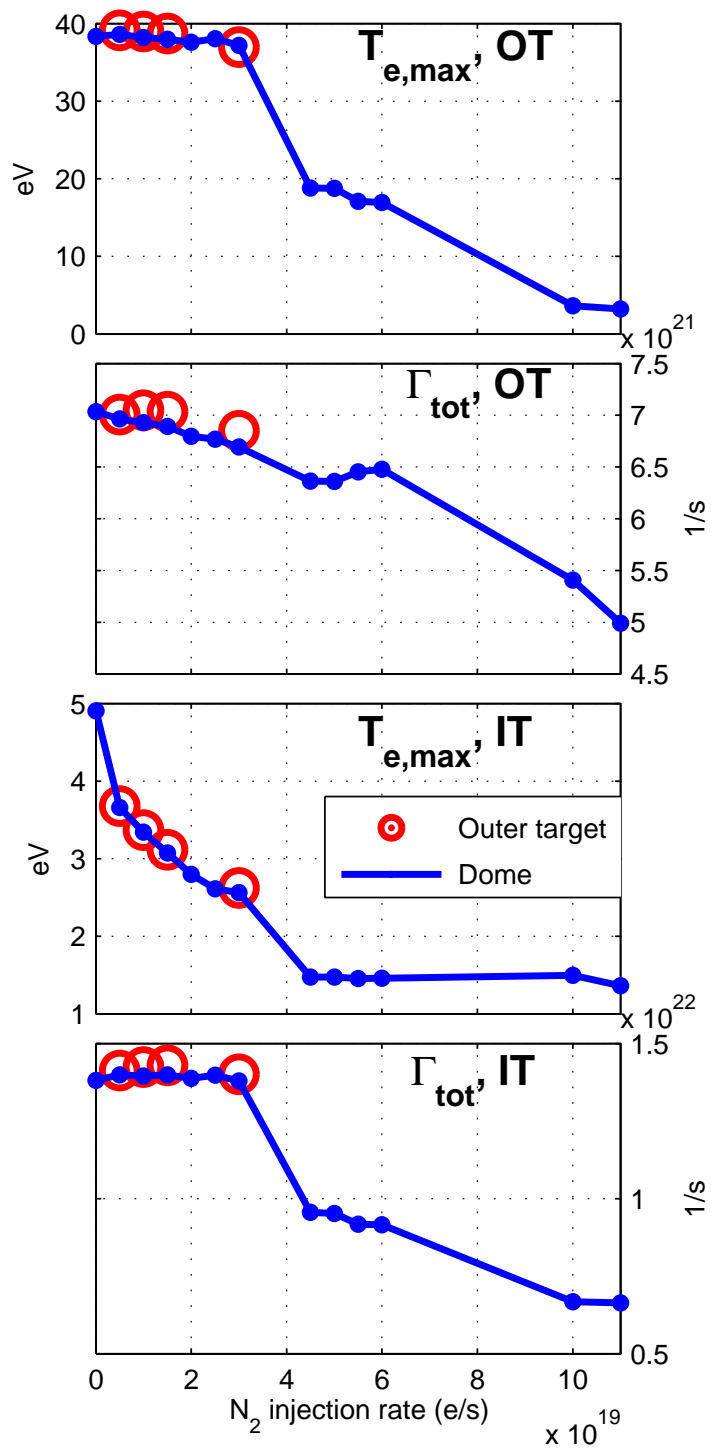

Figure 5 